\title{
Anomalous Muscles of the Wrist Encountered During Endoscopic Carpal Tunnel Surgery
}

\author{
Se-Hyuck Park, M.D. \\ Department of Neurosurgery, Gangdong Sacred Heart Hospital, Hallym University Collge of Medicine, Seoul, Korea
}

Objective : Anomalous muscles of the wrist are infrequently encountered during carpal tunnel surgery. Anatomic variants of the palmaris longus (PL), flexor digitorum superficialis, lumbricalis and abductor digiti minimi (ADM) have been reported but are usually clinically insignificant. Anomalies of the wrist muscles, encountered during endoscopic carpal tunnel surgery have rarely been described. I conducted this study to evaluate muscular anomalies of the volar aspect of the wrist, encountered during endoscopic carpal tunnel surgery.

Methods : I studied a consecutive series of 1235 hands in 809 patients with carpal tunnel syndrome who underwent single-portal endoscopic carpal tunnel release (ECTR) from 2002 to 2014. Nine hundred seventy-three hands in 644 patients who had minimal 6-month postoperative follow-up were included in the study. The postoperative surgical outcome was assessed at least 6 months after surgery.

Results : In eight patients, anomalous muscles were found under the antebrachial fascia at the proximal wrist crease and superficial to the ulnar bursa, passing superficial to the transverse carpal ligament. Those anomalous muscles were presumed to be variants of the PL or accessory ADM muscle, necessitating splitting and retraction to enter the carpal tunnel during the ECTR procedure. Other muscle anomalies were not seen within the carpal tunnel on the endoscopic view. The surgical outcome for all eight wrists was successful at the 6-month postoperative follow-up.

Conclusion : Carpal tunnel surgeons, especially those using an endoscope should be familiar with unusual findings of anomalous muscles of the wrist because early recognition of those muscles can contribute to avoiding unnecessary surgical exploration and unsuccessful surgical outcomes.

Key Words : Carpal tunnel syndrome · Endoscopy · Variants.

\section{INTRODUCTION}

Anomalies of muscles of the wrist and hand are infrequently encountered on magnetic resonance imaging or during carpal tunnel surgery ${ }^{18,23,32,34)}$. Anomalous muscles such as ana- tomic variants of the palmaris longus (PL), flexor digitorum superficialis (FDS), lumbricalis and abductor digiti minimi (ADM) have been reported ${ }^{2,6,7,10,11,15,18,32-34)}$. Porsch and Marcortte reported the incidence of anomalous muscle responsible for carpal tunnel syndrome (CTS) is less than $1.5 \%$ of cases

\footnotetext{
- Received : May 11, 2018 •Revised : June 26, 2018 •Accepted : June 29, 2018

- Address for reprints : Se-Hyuck Park, M.D.

Department of Neurosurgery, Gangdong Sacred Heart Hospital, Hallym University Collge of Medicine, 150 Seongan-ro, Gangdong-gu, Seoul 05355, Korea Tel : +82-2-2224-2238, Fax : +82-2-473-7387, E-mail : sehyuck@hallym.or.kr
}

This is an Open Access article distributed under the terms of the Creative Commons Attribution Non-Commercial License (http://creativecommons.org/licenses/by-nc/4.0) which permits unrestricted non-commercial use, distribution, and reproduction in any medium, provided the original work is properly cited. 
but the true incidence of anomalous muscle causing compressive neuropathy at the wrist is not known ${ }^{21}$. Anomalous muscles are usually clinically insignificant and symptomatic anomalies are rare but understanding the clinical relevance of anomalous muscles is important to avoid confusion concerning unexpected findings during surgery and to achieve good surgical outcomes. Anomalies of muscles of the wrist, encountered during endoscopic carpal tunnel surgery have been rarely described. We conducted this study to evaluate muscular anomalies of the volar aspect of the wrist, those may be encountered during endoscopic carpal tunnel release (ECTR).

\section{MATERIALS AND METHODS}

I obtained Institutional Review Board of the Gangdong Sacred Heart Hospital for this study (IRB File No: 2018-04-006). We conducted a retrospective study of a consecutive series of 1235 hands in 809 patients with CTS who underwent singleportal ECTR with a subligamentous and extrabursal approach, using a forward-facing knife by a same surgeon (S.H.P.) from 2002 to 2014. Nine hundred seventy-three hands in 644 patients who had minimal 6-month postoperative follow-up were included in the study. Five hundred seventy-nine patients were female and 65 patients were male. The mean age was 53.5 years (range, 20-82). The postoperative surgical outcome was assessed at least 6 months after surgery on the basis of subjective satisfaction, and the motor and sensory functions of the hand. The patient's subjective satisfaction was evaluated on the basis of improvement of preoperative symptoms and resolution of postoperative pillar pain. Motor assessments included grip and key pinch strengths measured with hydraulic grip and hydraulic pinch dynamometers, respectively. Sensory assessment included touch threshold with the Semmes-Weinstein monofilament and static two-point discrimination with the Mackinnon-Dellon Disk-Criminator.

\section{Surgical technique of ECTR}

ECTR was carried out under monitored anesthetic care using intravenous injection of propofol without endotracheal intubation or general anesthesia, using a laryngeal mask device. After exsanguination of the arm and inflation of a tourniquet up to $250 \mathrm{~mm} \mathrm{Hg}, 1.0-\mathrm{cm}$ transverse incision was made along the proximal wrist crease on the ulnar side of the PL tendon.
Subcutaneous dissection exposes the antebrachial fascia under the surgical loupe. A transverse incision was made on the antebrachial fascia, exposing the underlying ulnar bursa. When anomalous muscle under the antebrachial fascia was found, the muscle was split and retracted to expose the underlying ulnar bursa enveloping the flexor tendons. Subligamentous dissection towards the third or fourth finger was carried out to reflect the synovial tissue from the undersurface of the transverse carpal ligament (TCL) and to create a space for the endoscopic device using a synovial elevator. A washboard texture could be felt in the undersurface of the TCL with the elevator and its tip could be felt on the palm as it passed the distal part of the TCL. A slotted cannula was introduced into the carpal tunnel and a rigid $25^{\circ}$-angled $2.7-\mathrm{mm}$ endoscope was inserted through the cannula. A manifestation of reduced transillumination could be observed in the palm while the endoscope was under the TCL. White transversely running fibers of the TCL and a fat pad distal to the distal edge of the TCL could be seen on the endoscopic view. After ensuring that there were no intervening structures in the operative field, the TCL was incised proximally to distally with a forward-facing knife. After the TCL had been completely cut out, both an escape of palmar fat into the carpal tunnel and retraction of cut edges of the TCL out of the endoscopic view could be observed, as well as increased transillumination through the palm. A right-angled probe was used to palpate the divided TCL. The tourniquet was then released. Wound closure was accomplished in layers following hemostasis and a bulky compressive dressing was applied.

\section{RESULTS}

Among 973 wrists in 644 patients, anomalous muscles were found in eight wrists in eight patients. Two patients underwent bilateral simultaneous ECTR but there were no patients with anomalous muscles in both wrists. The eight patients comprised seven women and one man, and the eight wrists included four from the right and four from the left sides.

All anomalous muscles were located under the antebrachial fascia at the proximal wrist crease, crossing the wrist in an oblique or longitudinal direction and superficial to the ulnar bursa (Fig. 1A and B). Those anomalous wrist muscles lying deep to the antebrachial fascia and superficial to the ulnar 
bursa, passing superficial to the TCL were presumed to be variants of the PL tendon or accessory ADM muscle, necessitating the additional procedure of splitting and retracting those anomalous muscles to enter the carpal tunnel (Fig. 1C). Other anomalies of muscles of the wrist were not seen within the carpal tunnel on endoscopic view (Fig. 1D). The surgical outcome for all eight wrists was successful at the 6-month postoperative follow-up. All patients were satisfied with the results, and the motor and sensory functions of the hands had recovered at the 6-month follow-up as follows. Preoperative grip strength $(20.01 \pm 8.47 \mathrm{~kg})$ had recovered 6 months postop-

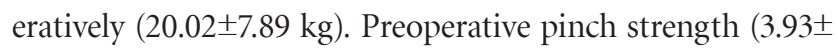
$1.38 \mathrm{~kg})$ had improved 6 months postoperatively $(4.70 \pm 1.54$ $\mathrm{kg})$. Both preoperative touch threshold (2.02 \pm 1.17$)$ and twopoint discrimination $(4.07 \pm 1.80)$ had improved at the 6-month follow-up to $1.38 \pm 0.49$ and $2.73 \pm 0.78$, respectively.
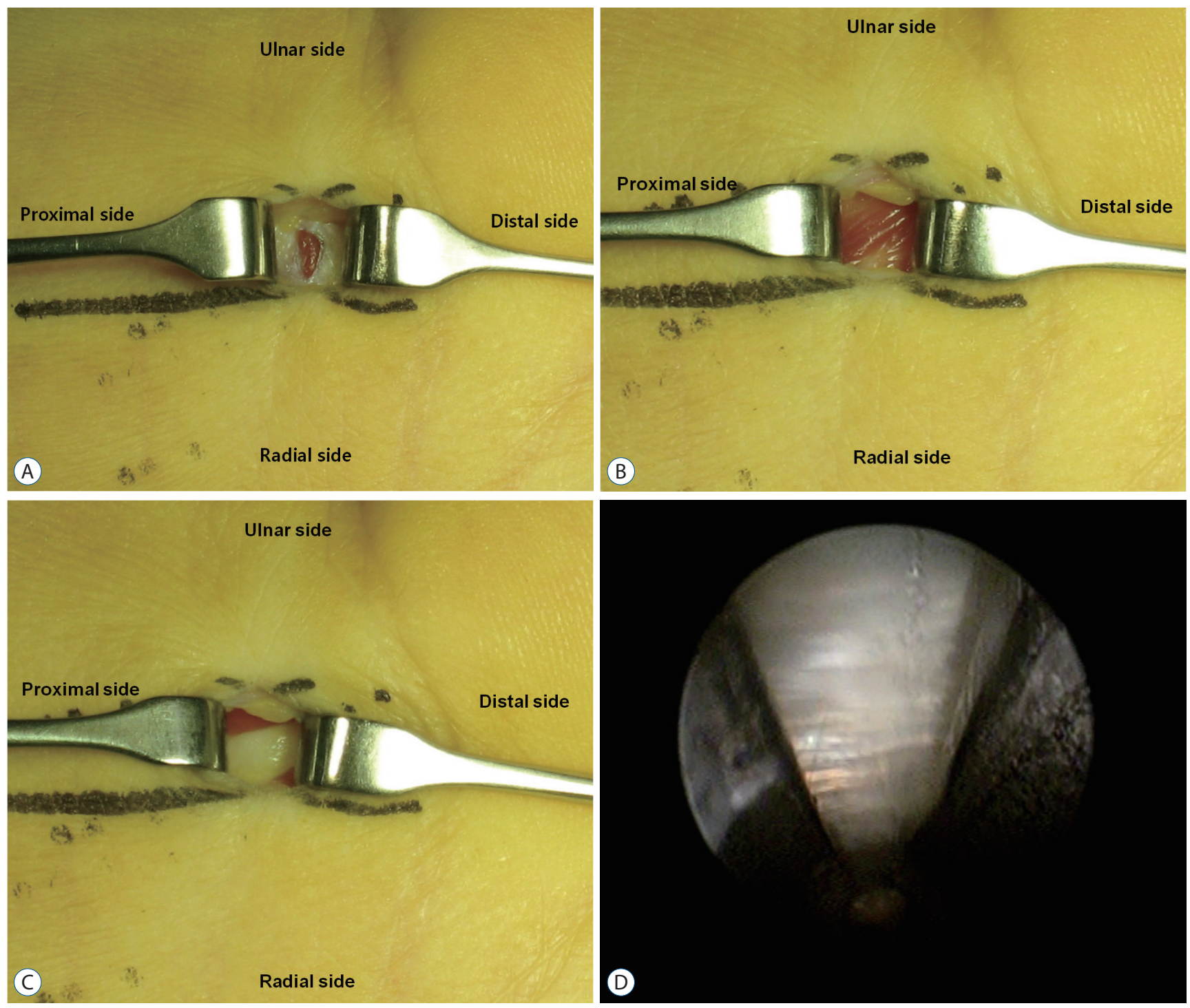

Fig. 1. Operative findings of the right wrist with an anomalous muscle in a 51-year-old man with carpal tunnel syndrome. A : Exposure of the anomalous muscle through a transverse incision of the antebrachial fascia. B : More exposure of the anomalous muscle in an oblique direction after retraction of the incised antebrachial fascia. C : Exposure of the underlying ulnar bursa surrounding the flexor tendons after retraction of the anomalous muscle. D: The undersurface of the transverse carpal ligament without any abnormal structures in the carpal tunnel on the endoscopic view. 


\section{DISCUSSION}

Carpal tunnel syndrome is the most common entrapment neuropathy in the upper extremity. Endoscopic carpal tunnel release is becoming widely used as a minimally invasive surgical technique with the advantages of less postoperative pain, faster improvement of grip and pinch strength and earlier return to work, compared with an open technique ${ }^{3,16,19,31)}$. However, anomalies of the muscles, exposed after an incision of the antebrachial fascia and before entrance into the carpal tunnel during an endoscopic procedure may embarrass inexperienced surgeons. Preoperative recognition of those anomalous muscles at the wrist can be challenging. Therefore, we need to know the anatomic variations of wrist muscles, which may be encountered during carpal tunnel surgery. However, the endoscopic view is too limited and restricted to observe all the intracarpal contents and our subligamentous and extrabursal approach has difficulty to discover intrabursal pathologies so that the incidence of anomalies of muscles in the wrist can be underestimated.

Anomalous muscles of the volar aspect of the wrist have been described in the literature including anatomic variations of the PL muscle, accessory FDS muscle, proximal origin of lumbrical muscle, accessory ADM muscle and the variant transverse carpal muscle $e^{2,6,7,10,11,15,18,32-34)}$. Those variants of the wrist muscles may be associated with symptoms related to compressive neuropathy of the median or ulnar nerve but symptomatic anomalies are rare.

The PL muscle is a forearm muscle with a short proximal muscular belly and a long distal tendon. It is the most variable muscle in the forearm and wrist and is associated with numerous anatomical anomalies ${ }^{9}$. The PL is located in the superficial layer of the anterior compartment of the forearm and originates from the medial epicondyle of the humerus as part of the common flexor tendon and inserts into the palmar aponeurosis. The PL is presumed to lie deep to the antebrachial fascia from origin to insertion and it may course along the suprafascial plane at the distal forearm, passing superficial to the $\mathrm{TCL}^{26)}$. Therefore, an anomalous PL muscle may be encountered under the antebrachial fascia at the wrist during an ECTR procedure, just like in my cases. Variants of the PL muscle include absent PL, multiple PL muscle bellies, reversed PL, entirely muscular PL, and palmaris profundus ${ }^{18,25,28)}$. Absence of the PL is the most common variant of PL and the prevalence is reported to range from $2 \%$ to $23.1 \%{ }^{12,24,27,30)}$. Since the PL muscle is used as an important topographical landmark for wrist or forearm surgery including endoscopic carpal tunnel surgery, and is also commonly utilized for tendon reconstruction surgery, preoperative identification of the presence or absence of the PL tendon is very important. Multiple PL muscle bellies include duplicated, bifid, bitendinous or digastric PL muscles. The digastric variant has a distal extra-muscle belly, sharing the same tendon, while the entirely muscular PL is a nontendinous variant ${ }^{1,18)}$. A reversed PL is an anatomical variation that is tendinous proximally and muscular distally, opposite to the pattern seen in the normal PL. Epifascial PL is an extremely rare anomaly, where the PL originates from the subcutaneous fascia and inserts on palmar aponeurosis $^{25)}$. The digastric, completely muscular, reversed PL and epifascial variants of the PL may reveal soft tissue masses with bluish skin discoloration at the wrist, which can be encountered at the wrist during the endoscopic carpal procedure $^{20,25)}$. The anomalous wrist muscles, which lay deep in the antebrachial fascia and superficial to the ulnar bursa, that were encountered in our series were presumed to be reversed or digastric variants of the PL tendon.

The palmaris profundus muscle is a rare variant of the PL and may be included in the duplicated PL muscle classification $^{18)}$. It originates from the palmar surface of the radius or the neighboring flexor muscles ${ }^{4,13)}$. The distal tendon passes through the carpal tunnel deep to the flexor retinaculum and inserts into the undersurface of the superficial palmar aponeurosis ${ }^{14,18)}$. It is uncertain whether the palmaris profundus is responsible for the symptoms of CTS but it is recommended to excise the palmaris profundus when it is encountered during a carpal tunnel release ${ }^{14,18)}$. A case of a palmaris profundus tendon, attached to the undersurface of the proximal TCL as an impeding structure during ECTR, necessitating the conversion of the procedure to an open technique has been reported $^{14)}$.

An accessory flexor digitorum superficialis muscle of the index finger is an unusual anomalous muscle of the wrist. It usually originates from the FDS tendon adjacent to the TCL and inserts into the second metacarpal bone, however, several other variants have been reported ${ }^{5,8,22,28)}$. An accessory FDS with more proximal extension of the muscle belly into the carpal tunnel may be encountered during carpal surgery. However, if the ulnar bursa is not violated during endoscopic 
surgery with a subligamentous and extrabursal approach, this accessory muscle can be difficult to detect because it is located within the ulnar bursa.

The lumbrical muscles normally originate from the radial side of the flexor digitorum profundus tendon, just distal to the carpal tunnel and insert into the extensor expansion near the metacarpophalangeal joint ${ }^{15,17,29)}$. However, anatomical variant of an abnormally proximal origin of the lumbrical muscle within the carpal tunnel may cause $\mathrm{CTS}^{17,29)}$. Because of its intrabursal location it cannot be seen when using an endoscopic subligamentous extrabursal approach.

Anomalous muscles in the hypothenar area occur less frequently. Of anomalous hypothenar muscles, variations of the ADM muscle are more frequent than those of the opponens and flexor digiti minimi muscles ${ }^{7,10,32)}$. The accessory ADM muscle originates variably from the flexor carpi ulnaris tendon, flexor retinaculum, distal PL tendon or forearm fascia and inserts into the ADM muscle or into the ulnar side of the proximal metacarpal base $e^{6,10,25,32)}$. If the accessory ADM muscle originates from distal PL tendon or the forearm fascia, it could be encountered deep to the antebrachial fascia and superficial to the ulnar bursa at the wrist during endoscopic carpal tunnel surgery. If the accessory ADM muscle is encountered in the operative field, it can be split and retracted to allow entry into the carpal tunnel for introduction of a cannula with a telescope.

The palmaris brevis muscle is known to originate from the flexor retinaculm and palmar aponeurosis and insert into the pisiform bone and hypothenar dermis, however, it may have a variable origin and insertion $^{18)}$. If the palmaris brevis muscle originates more proximally, it can be exposed through wrist incision and it may be mistakenly identified as an anomalous PL muscle such as an accessory or reversed $\mathrm{PL}^{18)}$. However, as the proximally-originated palmaris brevis muscle lies superficial to the antebrachial fascia, splitting or retraction of the muscle is enough to expose the antebrachial fascia before entering the carpal tunnel.

\section{CONCLUSION}

Carpal tunnel surgeons especially those using an endoscope should be familiar with unusual findings of anomalous muscles of the wrist particularly anatomic variants of the PL or
ADM muscles because early recognition of those muscles can contribute to avoiding unnecessary surgical exploration and unsuccessful surgical outcomes. As anomalous muscles of the wrist are usually encountered as intraoperative incidental findings and are less symptomatic, proximal or distal exploration is rarely indicated to identify the origin and insertion of the variants.

\section{CONFLICTS OF INTEREST}

No potential conflict of interest relevant to this article was reported.

\section{INFORMED CONSENT}

This type of study does not require informed consent.

\section{- Acknowledgements}

The author thanks Soo-A Nam, RN at Gangdong Sacred Heart Hospital for her organization of the research data and writing assistance.

\section{References}

1. Backhouse KM, Churchill-Davidson D : Anomalous palmaris longus muscle producing carpal tunnel-like compression. Hand 7 : 22-24, 1975

2. Basu SS, Hazary $S$ : Variations of the lumbrical muscles of the hand. Anat Rec 136 : 501-504, 1960

3. Chen L, Duan X, Huang X, LV J, Peng K, Xiang Z : Effectiveness and safety of endoscopic versus open carpal tunnel decompression. Arch Orthop Trauma Surg 134 : 585-593, 2014

4. Chou HC, Jeng H, Ko TL, Pai MH, Chang CY, Wu CH : Variant palmaris profundus enclosed by an unusual loop of the median nerve. J Anat 199 (Pt 4) : 499-500, 2001

5. Christensen $S$ : Anomalous muscle belly of the flexor digitorum superficialis in two generations. Hand $9: 162-164,1977$

6. De Smet $\mathrm{L}$ : Median and ulnar nerve compression at the wrist caused by anomalous muscles. Acta Orthop Belg 68 : 431-438, 2002

7. Dodds GA 3rd, Hale D, Jackson WT : Incidence of anatomic variants in Guyon's canal. J Hand Surg Am 15 : 352-355, 1990

8. Elias LS, Schulter-Ellis FP : Anomalous flexor superficialis indicis: two case reports and literature review. J Hand Surg Am 10 : 296-299, 1985 
9. Fatah MF : Palmaris profundus of Frohse and Fränkel in association with carpal tunnel syndrome. J Hand Surg Br 9 : 142-144, 1984

10. Jeffery AK : Compression of the deep palmar branch of the ulnar nerve by an anomalous muscle. Case report and review. J Bone Joint Surg Br 53 : 718-723, 1971

11. Kaplan EB : Muscular and tendinous variations of the flexor superficialis of the fifth finger of the hand. Bull Hosp Joint Dis 30 : 59-67, 1969

12. Kawashima T, Kikushima S, Yokota E, Ohkubo F, Yamana $Y$, Sato F, et al. : A case of an accessory palmaris longus muscle and a duplicate palmaris longus muscle with special reference to their nerve supply-morphologic significance of a common innervation trunk. Okajimas Folia Anat Jpn 79 : 75-81, 2002

13. Lahey MD, Aulicino PL : Anomalous muscles associated with compression neuropathies. Orthop Rev 15 : 199-208, 1986

14. McClelland WB Jr, Means KR Jr : Palmaris profundus tendon prohibiting endoscopic carpal tunnel release: case report. J Hand Surg Am 37 : 695-698, 2012

15. Mehta HJ, Gardner WU : A study of lumbrical muscles in the human hand. Am J Anat 109 : 227-238, 1961

16. Michelotti B, Romanowsky D, Hauck RM : Prospective, randomized evaluation of endoscopic versus open carpal tunnel release in bilateral carpal tunnel syndrome: an interim analysis. Ann Plast Surg 73 Suppl 2 : S157-S160, 2014

17. Middleton WD, Kneeland JB, Kellman GM, Cates JD, Sanger JR, Jesmanowicz $A$, et al. : MR imaging of the carpal tunnel: normal anatomy and preliminary findings in the carpal tunnel syndrome. AJR Am J Roentgenol 148 : 307-316, 1987

18. Park MJ, Namdari S, Yao J : Anatomic variations of the palmaris longus muscle. Am J Orthop (Belle Mead NJ) 39 : 89-94, 2010

19. Park SH, Cho BH, Ryu KS, Cho BM, Oh SM, Park DS : Surgical outcome of endoscopic carpal tunnel release in 100 patients with carpal tunnel syndrome. Minim Invasive Neurosurg 47 : 261-265, 2004

20. Polesuk BS, Helms CA : Hypertrophied palmaris longus muscle, a pseudomass of the forearm: MR appearance--case report and review of the literature. Radiology 207 : 361-362, 1998

21. Posch JL, Marcotte DR : Carpal tunnel syndrome: an analysis of 1,201 cases. Orthop Rev 5 : 25-35, 1976

22. Probst $C E$, Hunter JM : A digastric flexor digitorum superficialis. Bull Hosp Joint Dis 36 : 52-57, 1975

23. Ragoowansi $R$, Adeniran A, Moss AL : Anomalous muscle of the wrist. Clin Anat 15 : 363-365, 2002

24. Sebastin SJ, Puhaindran ME, Lim AY, Lim IJ, Bee WH : The prevalence of absence of the palmaris longus--a study in a Chinese population and a review of the literature. J Hand Surg Br 30 : 525-527, 2005

25. Sookur PA, Naraghi AM, Bleakney RR, Jalan R, Chan O, White LM : Accessory muscles: anatomy, symptoms, and radiologic evaluation. Radiographics $28:$ 481-499, 2008

26. Stecco C, Lancerotto L, Porzionato A, Macchi V, Tiengo C, Parenti A, et al. : The palmaris longus muscle and its relations with the antebrachial fascia and the palmar aponeurosis. Clin Anat 22 : 221-229, 2009

27. Thompson NW, Mockford BJ, Cran GW : Absence of the palmaris longus muscle: a population study. Ulster Med J 70 : 22-24, 2001

28. Timins ME : Muscular anatomic variants of the wrist and hand: findings on MR imaging. AJR Am J Roentgenol 172 : 1397-1401, 1999

29. Touborg-Jensen A : Carpal-tunnel syndrome caused by an abnormal distribution of the lumbrical muscles. Case report. Scand J Plast Reconstr Surg $4: 72-74,1970$

30. Troha F, Baibak GJ, Kelleher JC : Frequency of the palmaris longus tendon in North American Caucasians. Ann Plast Surg 25 : 477-478, 1990

31. Vasiliadis HS, Georgoulas P, Shrier I, Salanti G, Scholten RJ : Endoscopic release for carpal tunnel syndrome. Cochrane Database Syst Rev (1) : CD008265, 2014

32. Zeiss J, Guilliam-Haidet $L$ : MR demonstration of anomalous muscles about the volar aspect of the wrist and forearm. Clin Imaging 20 : 219-221, 1996

33. Zeiss J, Jakab E, Khimji T, Imbriglia J : The ulnar tunnel at the wrist (Guyon's canal): normal MR anatomy and variants. AJR Am J Roentgenol 158 : 1081-1085, 1992

34. Zeiss J, Jakab E : MR demonstration of an anomalous muscle in a patient with coexistent carpal and ulnar tunnel syndrome. Case report and literature summary. Clin Imaging 19 : 102-105, 1995 